\title{
Obesity-associated miR-27a upregulation promotes hepatocellular carcinoma metastasis through suppressing SFRPI
}

This article was published in the following Dove Press journal:

OncoTargets and Therapy

Yang Chen'
Fan Zhang'
Yawei Zhao'
Kan He'
Xiao Zheng'
Yue Pan'
Dan Shao'
Pei Shang
Yongsheng Yang'
Dan Zhang'
Yingjun Xie'
Xiaoxiao Yao'
Li Chen ${ }^{2}$
Jing Li'
Xuewen Zhang'
'Department of Hepatobiliary and
Pancreas Surgery, The Second Hospital
of Jilin University, Changchun I3004I,
China; 'Department of Pharmacology,
Nanomedicine Engineering Laboratory
of Jilin Province, College of Basic
Medical Sciences, Jilin University,
Changchun I3002I, China

Correspondence: Xuewen Zhang Department of Hepatobiliary and Pancreas Surgery, The Second Hospital of jilin University, No 218 Ziqiang Street, Nanguan District, Changchun I3004I, China

Tel +86 43I 88796222

Email zxw515@sohu.com

Jing Li

Department of Pharmacology,

Nanomedicine Engineering Laboratory

of jilin Province, College of Basic Medical

Sciences, Jilin University, No I26 Xinmin

Street, Chaoyang District, Changchun

|3002|, China

Tel +86 43I 856I 9799

Email lijing@jlu.edu.cn
Background: Obesity was a recognized risk factor for the development and progression of hepatocellular carcinoma (HCC). However, the effects and mechanisms by which obesity promotes HCC metastasis remain poorly understood.

Materials and methods: We cultured adipocyte induced by preadipocyte 3T3-L1 in vitro and established HCC metastasis model in obesity mouse in vivo to mimic the tumor microenvironment in obese status. The mechanisms underlying obesity-associated miR-27a upregulation promoting HCC metastasis were investigated.

Results: In this study, we showed that miR-27a was upregulated in adipocytes, obese mouse model and clinical samples, and the increased miR-27a level promoted migration and invasion in HCC cells, increased the number of metastasis nodes in obese mouse model, and was associated with poor clinical outcomes. Overexpressed secreted frizzled-related protein 1 in HCC cells and tissues significantly alleviated the upregulation of $\beta$-catenin and matrix metalloproteinase- 7 induced by high level of miR-27a. Meanwhile, the E-cadherin expression decreased and Vimentin expression increased, linking with high level of $\beta$-catenin in high-fat group.

Conclusion: Taken together, our results have elucidated the critical role of extracellular miR27a as a pro-metastatic factor in HCC and revealed that obesity-associated miR-27a upregulation promoted HCC metastasis through activated Wnt/ $\beta$-catenin signaling by suppressing secreted frizzled-related protein 1 . Our findings shed light on the novel mechanism underlying HCC metastasis and provided miR-27a as a promising target for obese liver cancer therapy.

Keywords: obesity, miR-27a, SFRP1, $\beta$-catenin, hepatocellular carcinoma

\section{Introduction}

Obesity has become an important public health challenge in recent decades all over the world. It is reported that there were more than 1.9 billion overweight adults in 2014, of whom over 600 million were obese. ${ }^{1}$ Moreover, obesity is recognized as a common risk factor for many chronic diseases, including type 2 diabetes, cardiovascular diseases, and cancers. ${ }^{2}$ Numerous experimental studies and meta-analyses have shown that obesity was directly associated with the risk of cancers, such as breast, endometrium, kidney, thyroid, esophagus, gastric cardia, pancreas, gallbladder, colon, liver, and so on. ${ }^{3}$ In addition, obesity can also lead to poor prognosis and result in higher cancer-related mortality. ${ }^{4-6}$

Hepatocellular carcinoma (HCC) is the fifth most common malignancy and the third leading cause of cancer-related deaths in the world. ${ }^{7}$ In recent years, although the treatment of HCC has improved, the prognosis of patients and the overall survival rate remain rather disappointing. ${ }^{8}$ Metastasis and local recurrence are responsible for 
the majority of HCC-related deaths, ${ }^{9}$ which have become the major obstacle in the treatment of patients with liver cancer. ${ }^{10}$ Most cases of HCC (70\%-90\%) development are associated with chronic liver disease backgrounds, such as the infection with hepatitis $B$ virus or hepatitis $C$ virus and alcoholic liver disease. ${ }^{11}$ While a substantial number of cases cannot be explained by these etiologies, HCC is increasingly being diagnosed among obese individuals. ${ }^{12}$ Many studies have indicated that the link between obesity and increased incidence of HCC has been globally verified. ${ }^{13-15}$ The molecular mechanisms underlying the higher risk of HCC in obesity are currently attributed to adipose tissue remodeling with proinflammatory adipokine secretion, cell growth stimulation by insulin and insulin-like growth factors, and liver lipotoxicity due to ectopic lipid accumulation. Moreover, diet, gut microbiome, and genetic factors are also involved. ${ }^{16-18}$ Studies mostly focused on obesity-related hepatocarcinogenesis up to now; however, the correlation between obesity and metastasis of HCC, as well as the molecular mechanisms are unknown.

In our previous work, we addressed a hypothesis that miR-27a, which is characteristically overexpressed during obesity, altered HCC microenvironment and promoted tumor progression. ${ }^{19,20}$ Herein, we first demonstrated that adipose tissue-secreted miR-27a is upregulated in obese liver cancer tissues. Next, we established the co-culture system of 3T3-L1 cells transfected with a miR-27a overexpress plasmid with HepG2 cells in vitro and confirmed that miR-27a promoted HCC cell migration and invasion under obesity condition. Finally, we established an animal model to investigate the effect of obesity-related upregulation of miR-27a on metastasis of HCC and revealed the mechanism in our current study. We found that miR-27a promoted migration and invasion of HCC cells and activated Wnt/ $\beta$-catenin signaling by suppressing secreted frizzled-related protein 1 (SFRP1) in vitro and in vivo. In addition, epithelial-mesenchymal transition (EMT) may induce obesity-related metastasis of HCC.

\section{Materials and methods Human tissue samples}

Twenty-five frozen HCC samples were obtained from the tissue specimen bank of our hospital. The clinical information of these cases was collected, which were divided into the normal $(n=15)$ and the obesity $(n=10)$ group based on the body mass index (BMI). ${ }^{21}$ This work was approved by the Ethics Committee of Jilin University and written informed consent was obtained from all patients for this study.

\section{Cell culture}

The mouse embryo 3T3-L1 cell line, the HCC cell line HepG2, and the mouse hepatoma cell line Hepa1-6 were obtained from the American Type Culture Collection (Manassas, VA, USA). The cells were grown in DMEM (Gibco, Carlsbad, CA, USA) with high glucose content, supplemented with $10 \%$ fetal bovine serum (FBS) (Gibco), 100 units $/ \mathrm{mL}$ penicillin, and $100 \mathrm{~g} / \mathrm{mL}$ streptomycin. All the cells were incubated at $37^{\circ} \mathrm{C}$ in a humidified chamber containing $5 \% \mathrm{CO}_{2}$. The primary antibodies against SFRP1 (rabbit, Cat. no. ab4193, Abcam, Cambridge, UK), $\beta$-catenin (rabbit, Cat. no. ab32572, Abcam), matrix metalloproteinase-7 (MMP7) (rabbit, Cat. no. ab5706, Abcam), Vimentin (rabbit, Cat. no. ab92547, Abcam), E-cadherin (mouse, Cat. no. ab76055, Abcam), and $\beta$-actin (mouse, Cat. no. sc-47778, Santa Cruz Biotechnology, Dallas, TX, USA) were diluted according to their instructions.

\section{Differentiation of 3T3-LI cell lines}

To acquire adipocyte simulating obese model in cells, 3T3-L1 preadipocytes were seeded in 24-well plates at a density of $1 \times 10^{5}$ per well and incubated when they reached about $80 \%$ confluence, and then the cells were differentiated by DMEM containing 10\% FBS and cocktail (MDI, $0.5 \mathrm{mM}$ isobutyl-1methylxanthine, $1 \mu \mathrm{M}$ dexamethasone, $10 \mu \mathrm{g} / \mathrm{mL}$ insulin) for 3 days. The medium was replaced by DMEM with $10 \%$ FBS and $10 \mu \mathrm{g} / \mathrm{mL}$ insulin, and the cells were incubated for another 3 days. The medium was then replaced with DMEM containing $10 \% \mathrm{FBS}$, and the cells continued to be cultured, waiting for a completion of differentiation. Adipocytes were only used for experiment when at least $95 \%$ of the cells showed an adipocyte phenotype with accumulation of lipid droplets.

\section{Transient transfection of plasmids}

The miR-27a expression plasmid, miR-27a inhibitor plasmid, SFRP1 overexpression plasmid, and the control vector were constructed by Shanghai GenePharma (Shanghai, China). The 3T3-L1 cells in exponential phase of growth were plated in plates at $2 \times 10^{5}$ cells $/ \mathrm{mL}$ and cultured for $16 \mathrm{~h}$, then transfected with the miR-27a expression plasmid, miR-27a inhibitor plasmid, and the control vector using Lipofectamine 2000 (Invitrogen, Carlsbad, CA, USA) according to the manufacturer's protocol. Successful and stable transfection was ensured by flow cytometry (Figure S1). The efficiency of transfection was examined by quantitative reverse transcription polymerase chain reaction (qRT-PCR). HepG2 cells transfected with the SFRP1 plasmid and the control vector in 
the same way, and the efficiency of transfection was detected by Western blot analysis.

\section{qRT-PCR analysis}

Total RNA was isolated from cells, tissues, serums and culture media with TRIzol reagent (Invitrogen). RNA was reverse transcribed into cDNA with TransScript FirstStrand cDNA synthesis supermix (Invitrogen), following the manufacturer's instructions. qRT-PCR was performed using a FastStart Universal STBR Green Master (Rox) from Roche (Basel, Switzerland). Primers of miR-27a and U6 were obtained from Ribobio (Guangzhou, China). The expression of miR-27a was normalized with U6 using the $2^{-\Delta \mathrm{Ct}}$ method. The miRNA expression levels were defined based on the threshold cycle $(\mathrm{Ct})$, and the relative expression levels were calculated as $2^{-([\mathrm{Ct} \text { of miR-27a] }-[\mathrm{Ct} \text { of U6]) }}$.

\section{Cell migration and invasion assays}

The transwell 24-well Boyden chamber (Corning, Corning, NY, USA) with $8.0 \mu \mathrm{m}$ pore size poly-carbonate membrane was used for the migration and invasion assay according to the manufacturer's protocol. For migration assay, 3T3-L1 cells were trypsinized and seeded in the lower chamber at a density of $1 \times 10^{5}$ cells/well. After cell differentiation, the medium was replaced with $600 \mu \mathrm{L}$ of complete medium while $1 \times 10^{5}$ HepG2 cells were seeded in the upper chamber in $100 \mu \mathrm{L}$ of serum-free medium and the migration during $12 \mathrm{~h}$ toward the lower chamber. Cells remaining at the upper surface of the membrane were removed with cotton swabs and cells on the lower surface of the membrane were fixed with 4\% paraformaldehyde, stained with crystal violet solution and counted in 5 randomly chosen high-power fields (100 magnification). Cell invasion assay was carried out similarly, except that $100 \mu \mathrm{L}$ of 1:8 DMEM-diluted Matrigel (Becton Dickinson, Franklin Lakes, NJ, USA) was added to each well at $37^{\circ} \mathrm{C}$ for $6 \mathrm{~h}$ before cells were seeded onto the membrane, followed by incubation for $12 \mathrm{~h}$.

\section{Western blot analysis}

The total proteins of cells and tissues were lysed in PIPA lysis buffer containing $10 \mathrm{mM}$ Tris- $\mathrm{HCl}, 0.25 \mathrm{M}$ sucrose, $10 \mathrm{mM} \mathrm{NaCl}, 1 \mathrm{mM}$ ethylenediaminetetraacetic acid, 1\% sodium dodecyl sulfate (SDS), and protease inhibitor cocktail (Roche), $\mathrm{pH}$ 7.5. The lysates were centrifuged at $12,000 \mathrm{~g}$ for $15 \mathrm{~min}$ at $4^{\circ} \mathrm{C}$ and the supernatant obtained was labeled as total protein extract. The protein concentration was determined by BCA protein assay kit (Beyotime Institute of Biotechnology,
Shanghai, China). Equal quantities of protein $(20 \mu \mathrm{g})$ were loaded onto $12 \%$ SDS polyacrylamidegel electrophoresis (SDS-PAGE) gels. The electrophoresis was carried out first at $80 \mathrm{~V}$ for $20 \mathrm{~min}$ and followed by $160 \mathrm{~V}$ for $60-85 \mathrm{~min}$. The separated proteins were transferred to polyvinylidene fluoride membranes (Bio-Rad Laboratories, Hercules, CA, USA). The membranes were blocked with $5 \%$ non-fat milk in Tris-buffered saline containing $0.05 \%$ Tween-20 for $\sim 2 \mathrm{~h}$ at room temperature and then were probed with primary antibodies against SFRP1 (1:250 dilution), $\beta$-catenin (1:5,000 dilution), MMP7 (1:1,000 dilution), Vimentin (1:1,000 dilution), E-cadherin (1:1,000 dilution), and $\beta$-actin (1:1,000 dilution) overnight at $4^{\circ} \mathrm{C}$. The membranes were then washed and incubated with the horseradish peroxidase (HRP)-conjugated goat anti-rabbit secondary antibody (1:5,000 dilution, Cat. no. SA00001-1; ProteinTech Group, Rosemont, IL, USA) or HRP-conjugated goat anti-mouse secondary antibody (1:5,000 dilution, Cat. no. SA00001-2; ProteinTech Group) at room temperature for $1 \mathrm{~h}$. Bands were visualized by an ECL Western blotting detection system (Tanon 4200, Shanghai, China) and analyzed by Quantity-one software (Bio-Rad).

\section{Establishment of obesity mouse model}

Male C57BL/6J mice (18-20 g, 6 weeks) were purchased from the Animal Research Center (Beijing, China) and kept in environment-controlled specific-pathogen-free room $\left(20^{\circ} \mathrm{C} \pm 2^{\circ} \mathrm{C}\right.$, humidity of $40 \%-60 \%, 12$-h light/dark cycle) following Chinese government guidelines. All mice were given free access to food and water throughout the study. All animal experimental protocols were approved by the Institutional Animal Care and Use Committee of Jilin University. The experimental manipulation of mice was conducted in accordance with the National Institute of Health Guide for the Care and Use of Laboratory Animals and the approval of the Scientific Investigation Board of Science and Technology of Jilin Province (Changchun, China).

Mice were randomly divided into 2 groups and fed either a control diet containing 10\% kcal fat (D12450B, Research Diets, New Brunswick, NJ, USA) or high-fat (HF) diet containing 60\% kcal fat (D12492i, Research Diets). During the experimental period, their body weights were measured twice per week. After 10 weeks, 6 mice were selected from each group randomly and sacrificed. Serum was collected after centrifuging the whole blood at 3,000 rpm for $15 \mathrm{~min}$. Livers, lungs, and epididymal adipose tissues were resected and weighed. Half part of the organs was fixed in a $4 \%$ paraformaldehyde solution overnight and processed for 
paraffin sections followed by $\mathrm{H} \& \mathrm{E}$ stain and immunohistochemistry (IHC) stain, and the other half was stored in $-80^{\circ} \mathrm{C}$ immediately for qRT-PCR and Western blot analysis.

\section{Establishment of HCC metastasis mouse model}

After the obesity model was established, mice of both groups received the surgery for orthotopic transplantation. Mice were anesthetized with a mixture consisting of ketamine at $100 \mathrm{mg} / \mathrm{kg}$ (Heng Rui, China) and xylazine at $25 \mathrm{mg} / \mathrm{kg}$ (Sigma-Aldrich, St Louis, MO, USA) via intraperitoneal injection and a small subxiphoid midline incision was made to expose the left lateral lobe of the liver. Hepa1- 6 cells $\left(5 \times 10^{5}\right.$ in $50 \mu \mathrm{L}$ PBS medium with 50\% Matrigel) then were injected into the left lateral lobe. Following the injection, the liver surface at the site of needle tract should be covered with a drop of matrigel (Becton Dickinson) to avoid any possible early peritoneal seeding of the HCC cells. After 20 days, the mice were sacrificed. Lungs, livers, and epididymal adipose tissues were dissected and photographed. Serum was collected after centrifuging the whole blood at 3,000 rpm for $15 \mathrm{~min}$. At necropsy, the pulmonary metastasis colonies on the surface of the lungs were counted. Then, a half part of the organs was fixed in a $4 \%$ paraformaldehyde solution overnight and processed for paraffin sections followed by H\&E and IHC stains, and the other half was stored in $-80^{\circ} \mathrm{C}$ immediately for qRT-PCR and Western blot analysis.

\section{IHC}

The tissue sections were dewaxed in xylene and dehydrated in alcohol. Antigen retrieval was done by incubating the slides in citric acid buffer for $10 \mathrm{~min}$. After blocking with $3 \%$ hydrogen peroxide for $15 \mathrm{~min}$ and $5 \%$ bovine serum albumin, the slides were incubated overnight at $4{ }^{\circ} \mathrm{C}$ with the antibody against SFRP1 (1:800 dilution). After rinsing with PBS, the sections were incubated with a HRP anti-rabbit IgG antibody (Cowin Bioscience, Beijing, China) for $30 \mathrm{~min}$ at room temperature. The expression was visualized by diaminobenzidine tetrahydrochloride solution (DAB, Cowin Bioscience) staining. The sections were finally counterstained with hematoxylin for $5 \mathrm{~min}$. All slides were observed under a light microscope at a magnification of $100 \times$ and $400 \times$.

\section{Statistical analysis}

Statistical analysis was performed using Graphpad prism software 6.0 (GraphPad Software, La Jolla, CA, USA). All data were expressed as mean \pm SD. Statistical significance $(p<0.05)$ was evaluated using Student's $t$-test when only 2 groups were compared. If more than 2 groups were compared, evaluation of significance was performed using one-way analysis of variance, followed by Bonferroni's post hoc test. Kaplan-Meier survival method was performed to assess the prognostic significance of obesity and the difference of resulting curves was analyzed by the log-rank test. Spearman correlation coefficients were performed to determine the association between SFRP1 and miR-27a expression. A value with $p<0.05$ was considered as statistically significant.

\section{Results}

\section{miR-27a promotes migration and invasion of HCC cells in vitro}

Our previous study found that miR-27a promoted HCC cell proliferation through the downregulation of FOXO1.1 ${ }^{19}$ To determine whether miR-27a from adipose tissue influences the migration and invasion of the HCC, we established a co-culture transwell chamber system of differentiated adipocyte 3T3-L1 cells with HepG2 cells. As shown in Figure 1, qRT-PCR analysis confirmed that 3T3-L1 cells could secrete miR-27a into culture media and increase the miR-27a level of HepG2 cells. To enhance or eliminate the expression of miR-27a, we transfected miR-27a expression plasmids and miR-27a inhibitor plasmids into 3T3-L1 cells and obtained the expected results in 3T3-L1 adipocytes (lower chamber), culture medium, and HepG2 cells (up chamber) (Figure 1). Transwell assays showed that more HepG2 cells migrated or invaded into the lower chamber when they were co-cultured with 3T3-L1 cells (Figure 2). Moreover, overexpression of miR-27a in 3T3-L1 cells clearly enhanced the migration and invasion of HepG2 cells, whereas the opposite result was observed in the miR-27a inhibitor group.

\section{miR-27a activates $W n t / \beta$-catenin signaling by suppressing SFRPI in vitro}

The Wnt/ $\beta$-catenin signaling pathway is involved in $\mathrm{HCC}$ cell migration and invasion. ${ }^{22}$ Several studies have shown that SFRP1, a member of Wnt inhibitors, is negatively regulated by miR-27a. ${ }^{23,24}$ Western blot showed that overexpression of miR-27a in 3T3-L1 cells clearly suppressed SFRP1 in HepG2 cells and that inhibition of miR-27a did the opposite (Figure 3C), which was consistent with previous reports. To determine whether SFRP1 participates in secretory miR-27a from adipocytes-driven migration and invasion of HCC cells, we overexpressed SFRP1 in HepG2 cells. It was shown that SFRP1 protein level in HepG2 cells 

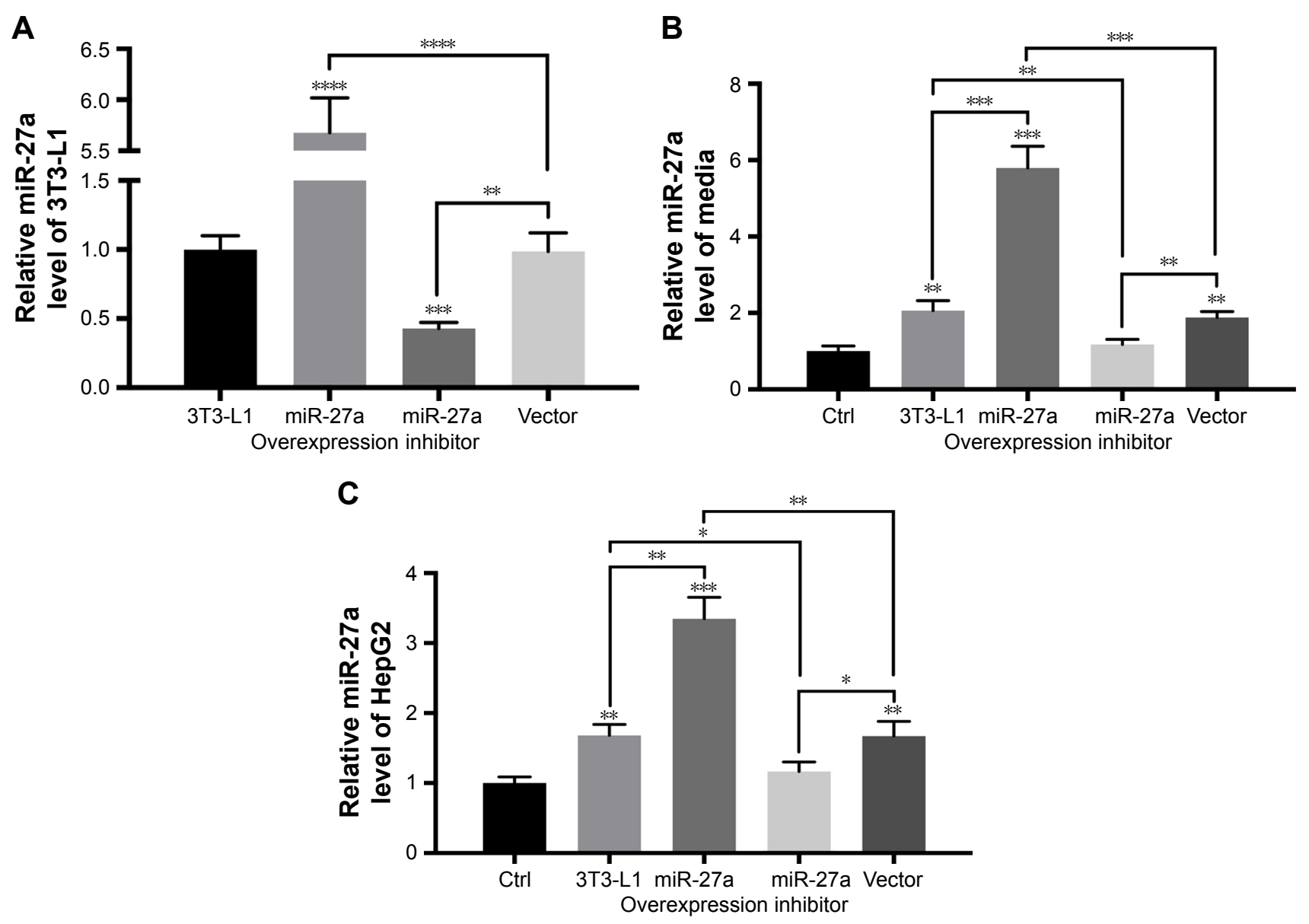

Figure I The establishment of co-culture transwell chamber system of differentiated adipocyte 3T3-LI cells with HepG2 cells.

Notes: (A) qRT-PCR analysis of miR-27a expression in 3T3-LI cells without transfection or transfected with miR-27a overexpression plasmid, miR-27a inhibitor plasmid, or empty vector after differentiation. (B, C) qRT-PCR analysis of miR-27a expression in cell culture medium (B) in the lower chamber or HepG2 cells (C) in the upper chamber alone, or with 3T3-LI, miR-27a overexpressing 3T3-LI, miR-27a inhibitor 3T3-LI and 3T3-LI transfected with empty vector co-culture. Each bar represents the mean \pm SD of 3 replicates. $* p<0.05$, ** $p<0.01$, *** $p<0.00$ I, and $* * * * p<0.000$ I.

Abbreviation: $q R T-P C R$, quantitative reverse transcription polymerase chain reaction.

increased three-fold after transfection (Figure 3A). But the migration and invasion of HepG2 cells co-cultured with 3T3-L1 in a miR-27a-enriched environment were substantially attenuated upon SFRP1 overexpression (Figure 3B). To further confirm the relationship between SFRP1 and the $\mathrm{Wnt} / \beta$-catenin signaling pathway in metastasis of HCC cells communication with adipocytes through secretory miR-27a, the related important protein levels were detected by western blot, including SFRP1, $\beta$-catenin, and its downstream MMP7. Overexpression of miR-27a in 3T3-L1 cells induced $\beta$-catenin and its downstream MMP7 expression level, and inhibition of miR-27a increased their expression (Figure 3C). Meanwhile, the induction of $\beta$-catenin and MMP7 by the secretory miR-27a from 3T3-L1 cells were reversed by overexpression of SFRP1 (Figure 3C), which confirmed the efficacy of SFRP1-inhibiting Wnt/ $\beta$-catenin signaling pathway. In addition, the expression of SFRP1 was increased through inhibiting the expression of miR-27a. Moreover, the expression of $\beta$-catenin and MMP-7 was also changed compared with control group. Taken together, our result suggested that secretory miR-27a could promote the metastasis of HCC cells through the degradation of SFRP1, of which could further activate $\mathrm{Wnt} / \beta$-catenin signaling pathway.

\section{miR-27a was upregulated in metastasis of HCC in vivo}

To investigate the profile of miR-27a in an obese individual in vivo, we established the HF diet-induced obese model of C57BJ mice. Mouse model of HCC metastasis was established by orthotopic syngeneic tumor transplantation in obese C57BJ mice mentioned previously. Twenty days after tumor transplantation, more visible metastasis nodes were observed on lung tissue compared with that of the control group (16.33 \pm 3.091 vs $34.17 \pm 6.149$; Figure 4A). 
A
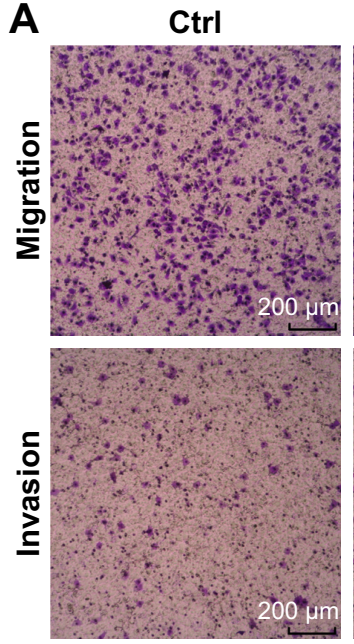

B
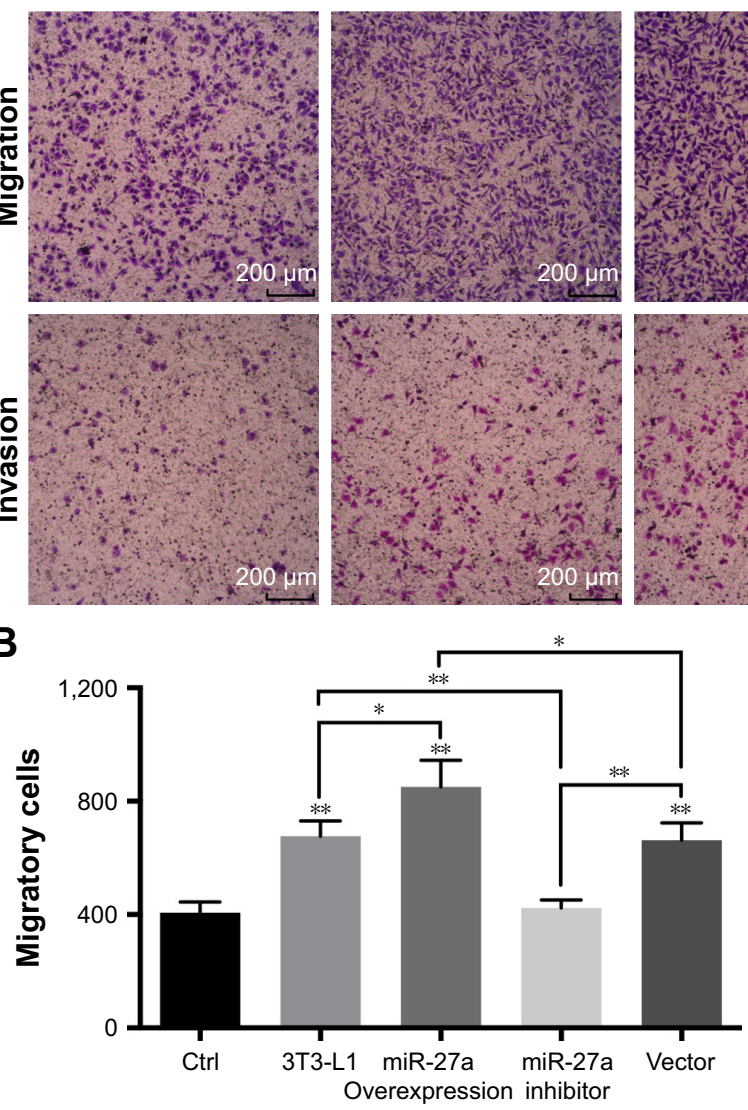

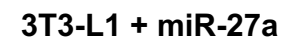

overexpression
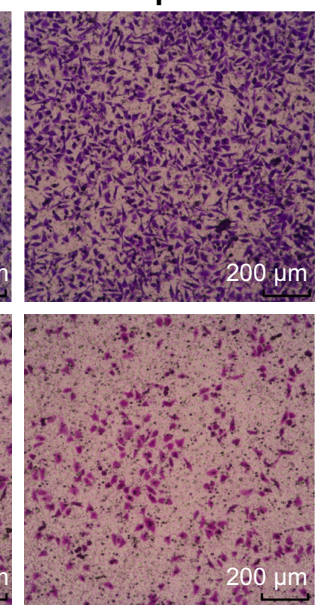

C

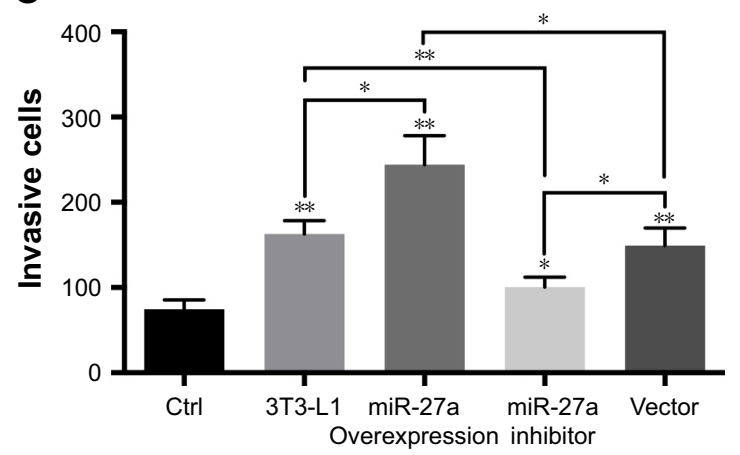

Figure 2 Secretory miR-27a promotes migration and invasion of $\mathrm{HCC}$ cells in vitro.

Notes: (A) Transwell assays were employed to evaluate the migratory and invasive ability of HepG2 cells under co-culture alone, or with 3T3-LI, miR-27a overexpressing 3T3-LI, miR-27a inhibitor 3T3-LI and 3T3-LI transfected with empty vector co-culture. The original magnification is $\times 40$, with a scale of I:200 $\mu$ m. (B, C) Quantitative results for the migratory and invasive ability of each group are shown as the number of cells. Each bar represents the mean \pm SD of 3 replicates. $* p<0.05$ and $* * p<0.0$ I.

Abbreviation: HCC, hepatocellular carcinoma.

The miR-27a levels of adipose tissue, liver, and serum were also significantly higher in the HF group than the control group (Figure 4B). To study the molecular mechanisms of the HCC metastasis under obesity condition, the protein expression of SFRP, $\beta$-catenin, and MMP7 were detected in liver tumor tissue. Western blot and IHC showed that SFRP1 expression was suppressed significantly compared with the control group (Figure 5A and B). Moreover, the protein levels of $\beta$-catenin and MMP7 were increased in HF group, which indicated the activation of $\mathrm{Wnt} / \beta$-catenin pathway in $\mathrm{HCC}$ may contribute distal metastasis. EMT-associated proteins Vimentin and E-cadherin were also detected to further elucidate the mechanisms of the HCC metastasis under obesity condition. Upregulation of Vimentin and downregulation of E-cadherin in protein expressions shown in HF group were compared with that of control group, which suggested that EMT was involved in HCC metastasis under obesity condition (Figure 5B). Collectively, these data demonstrated that
miR-27a was upregulated in metastasis of HCC in vivo and suppressed SFRP1. Activation of the Wnt/ $\beta$-catenin pathway, as well as induction of EMT may participate in HCC metastasis under obesity condition.

\section{SFRPI expression is negatively associated with the expression of miR-27a in human HCC tissues}

To analyze miR-27a-SFRP1 interactions in human HCC, we detected the expression of miR-27a using qRT-PCR and SFRP1 using Western blot in 25 frozen human HCC tissues (Figure 6A). The results showed that miR-27a was upregulated and that SFRP1 was downregulated in the obesity group, compared with the normal group. The inverse correlation between miR-27a and SFRP1 protein levels in human HCC were further illustrated using Spearman's correlation scatter plots (Figure 6B). Besides, we also detected the relationship between obesity and clinical outcome. 
A

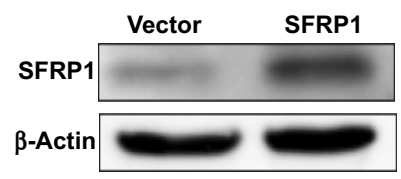

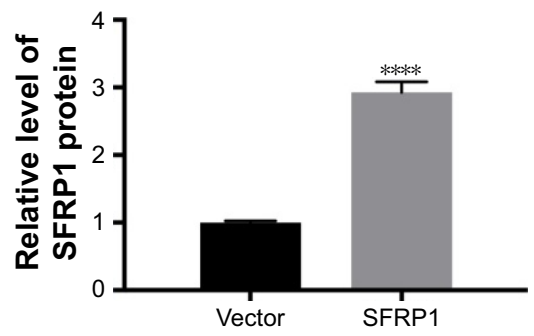

B HepG2 Vector

3T3-L1
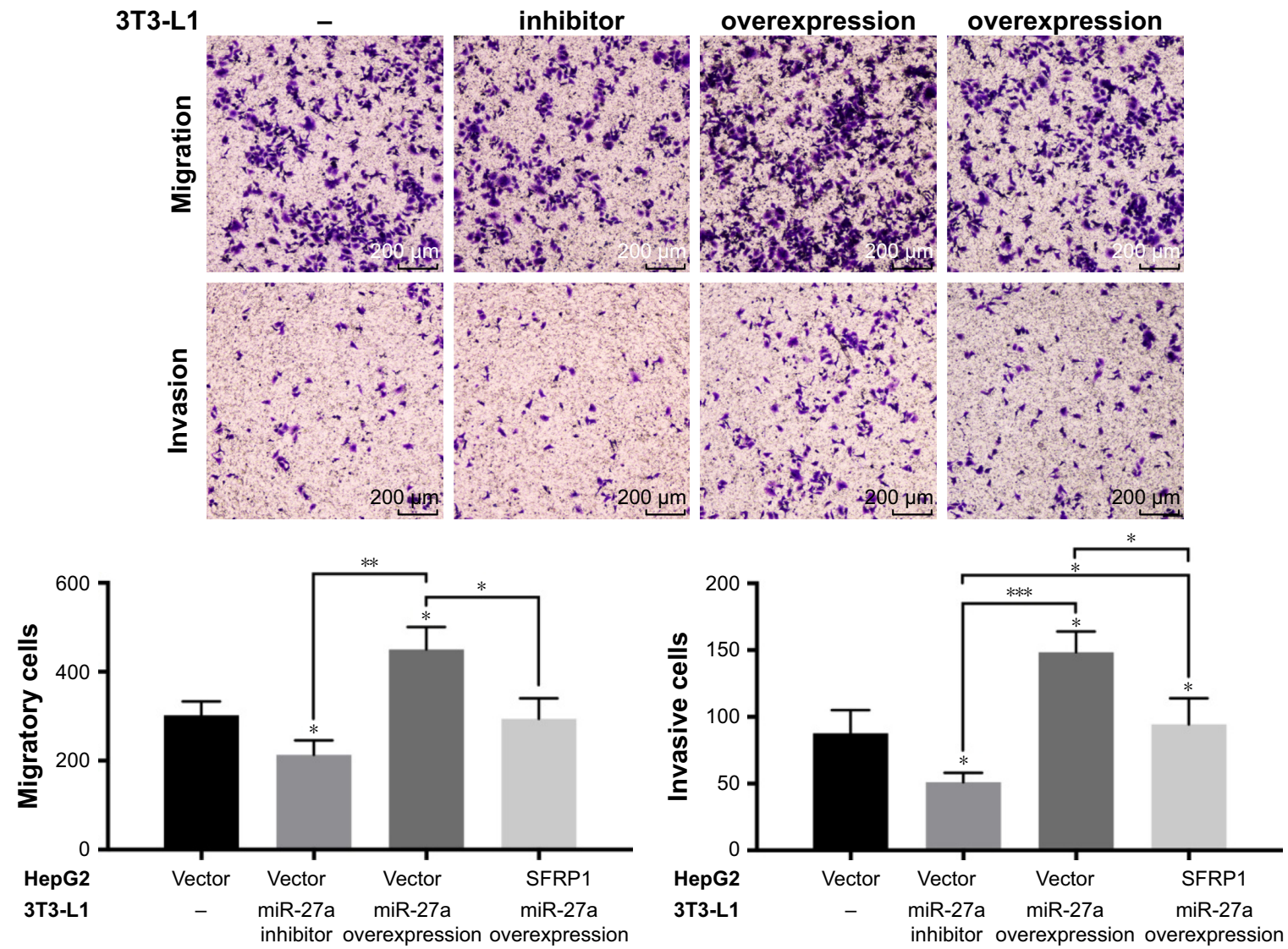

\section{C}

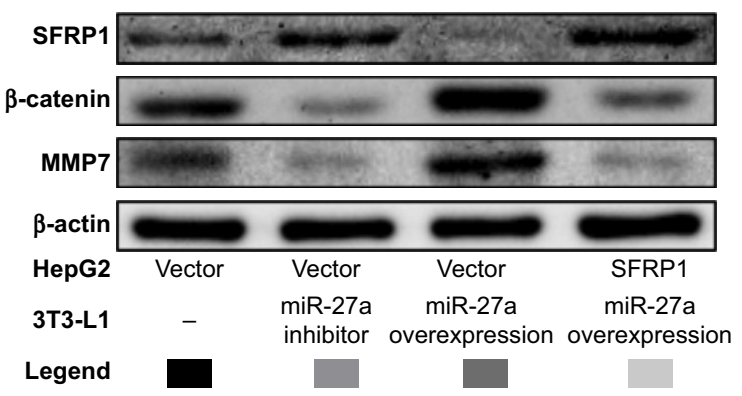

Figure 3 miR-27a activated wnt/ $\beta$-catenin signaling by suppressing SFRPI in vitro.

Notes: (A) Western blot analysis of SFRPI protein expression in HepG2 cells transfected with empty vector or SFRPI overexpressed plasmid. $\beta$-actin was used as an internal control. (B) Transwell assays were employed to evaluate the migratory and invasive ability of HepG2 cells transfected with empty vector or SFRPI-overexpressed plasmid under co-culture with 3T3-LI cells alone, miR-27a-inhibited 3T3-LI cells, or miR-27a-overexpressed 3T3-LI cells. The original magnification is $\times 40$, with a scale of I:200 $\mu$ m. Quantitative results for the migratory and invasive ability of each group are shown as the number of cells. (C) Protein expressions of SFRPI, $\beta$-catenin, MMP7 in HepG2 cells transfected with empty vector or SFRPI-overexpressed plasmid under co-cultured with 3T3-LI cells alone, miR-27a-inhibited 3T3-LI cells, or miR-27a-overexpressed 3T3$\mathrm{LI}$ cells. $\beta$-actin was used as an internal control. Each bar represents the mean $\pm \mathrm{SD}$ of 3 replicates. $* p<0.05, * * p<0.01$, *** $p<0.00 \mathrm{I}$, and $* * * * p<0.000 \mathrm{I}$.

Abbreviations: MMP7, matrix metalloproteinase-7; SFRPI, secreted frizzled-related protein I. 

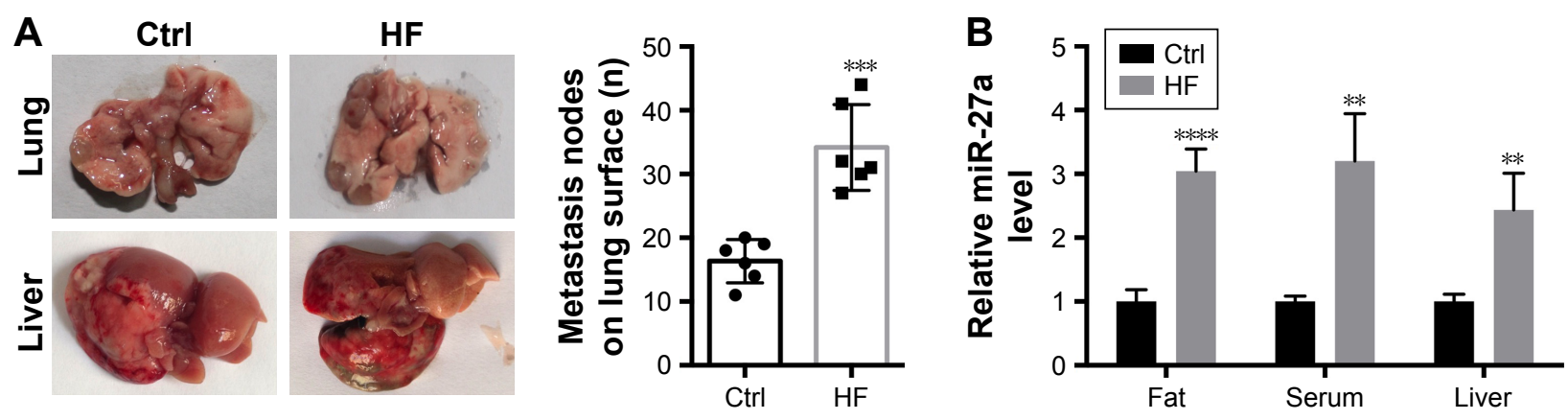

Figure 4 Obesity promoted HCC metastasis in vivo with upregulation of miR-27a.

Notes: (A) The livers and lungs were excised and metastasis nodes on lung surface were quantified in the control and the HF groups. (B) qRT-PCR analysis of miR-27a expression in adipose tissue, serum, liver, and lung from the control and the HF groups. Each bar represents the mean \pm SD of 3 replicates. $* * p<0.0 \mathrm{I}$, $* * * 0.00 \mathrm{I}$, and $* * * * p<0.0001$

Abbreviations: Ctrl, control; HCC, hepatocellular carcinoma; HF, high fat; qRT-PCR, quantitative reverse transcription polymerase chain reaction.

Follow-up data showed that the overall survival of HCC patients with high BMI were shorter than those with normal BMI (Figure 6C).

\section{Discussion}

Many studies revealed that obesity was one of the risk factors for various cancers, including HCC. ${ }^{3}$ Obesity not only increased the incidence of $\mathrm{HCC}$ but also resulted in impaired drug treatment efficacy and poor prognosis of HCC. ${ }^{16,18}$
However, the mechanism underlying obesity promoting the development and progression of liver cancer is unclear. Recently, the tumor microenvironment in association with endocrine modifications provided by the unique adipose tissue, promoting the initiation, proliferation, invasion, and metastatic progression of tumor, has been extensively discussed. Besides adipokine and cytokine (leptin, adiponectin, interleukin [IL]-6, tumor necrosis factor alpha, and IL-1 $\beta$ ), adipogenic microRNA became a new class of regulatory
A
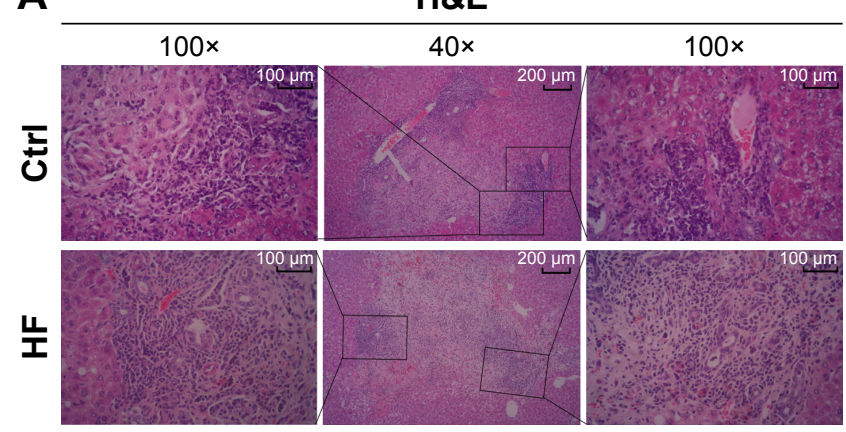

B

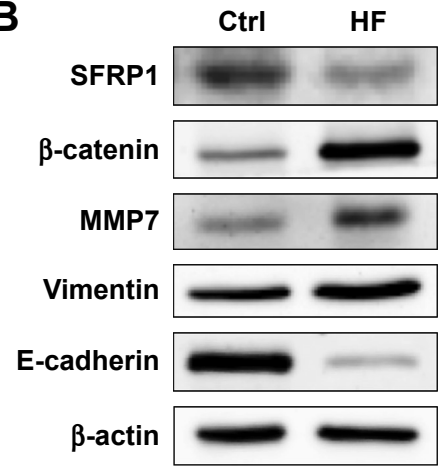

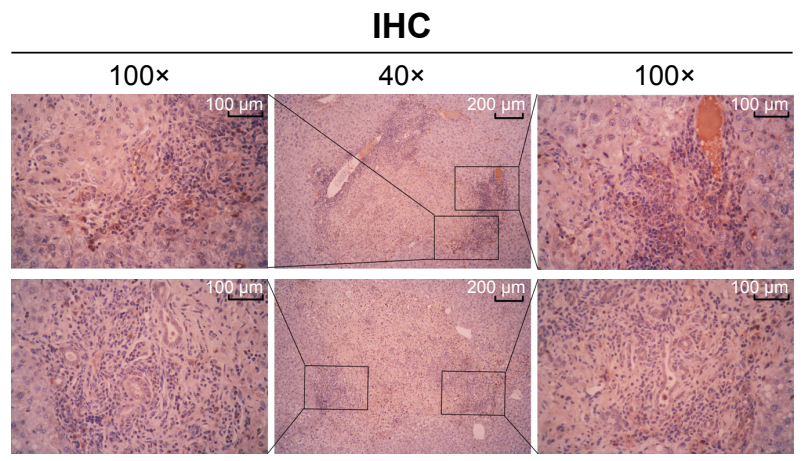

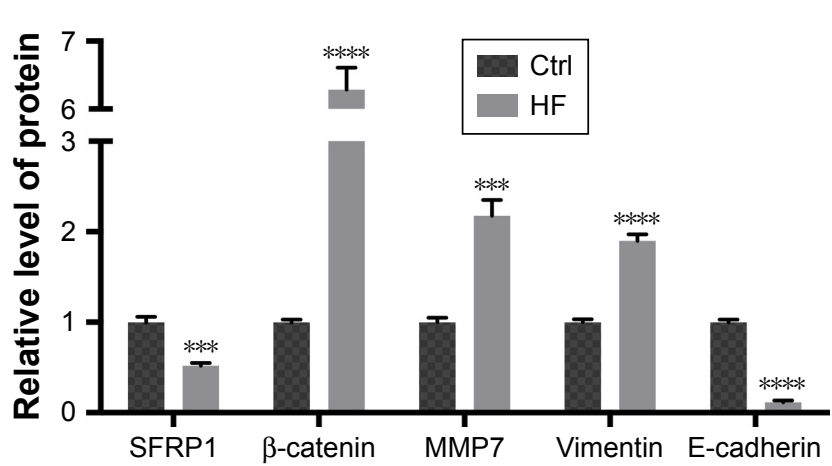

Figure $5 \mathrm{Wnt} / \beta$-catenin signaling was activated in $\mathrm{HCC}$ metastasis of obesity in vivo.

Notes: (A) H\&E staining and SFRPI IHC staining of liver in the control and the HF groups. (B) Western blot analysis of SFRPI, $\beta$-catenin, MMP7, vimentin, and E-cadherin protein expression in tumor foci of liver from the control and the HF groups. $\beta$-actin was used as an internal control. Each bar represents the mean \pm SD of 3 replicates. $* * * p<0.00$ I, and $* * * * p<0.0001$.

Abbreviations: Ctrl, control; HCC, hepatocellular carcinoma; HF, high fat; IHC, immunohistochemistry; MMP7, matrix metalloproteinase-7; SFRPI, secreted frizzledrelated protein I. 

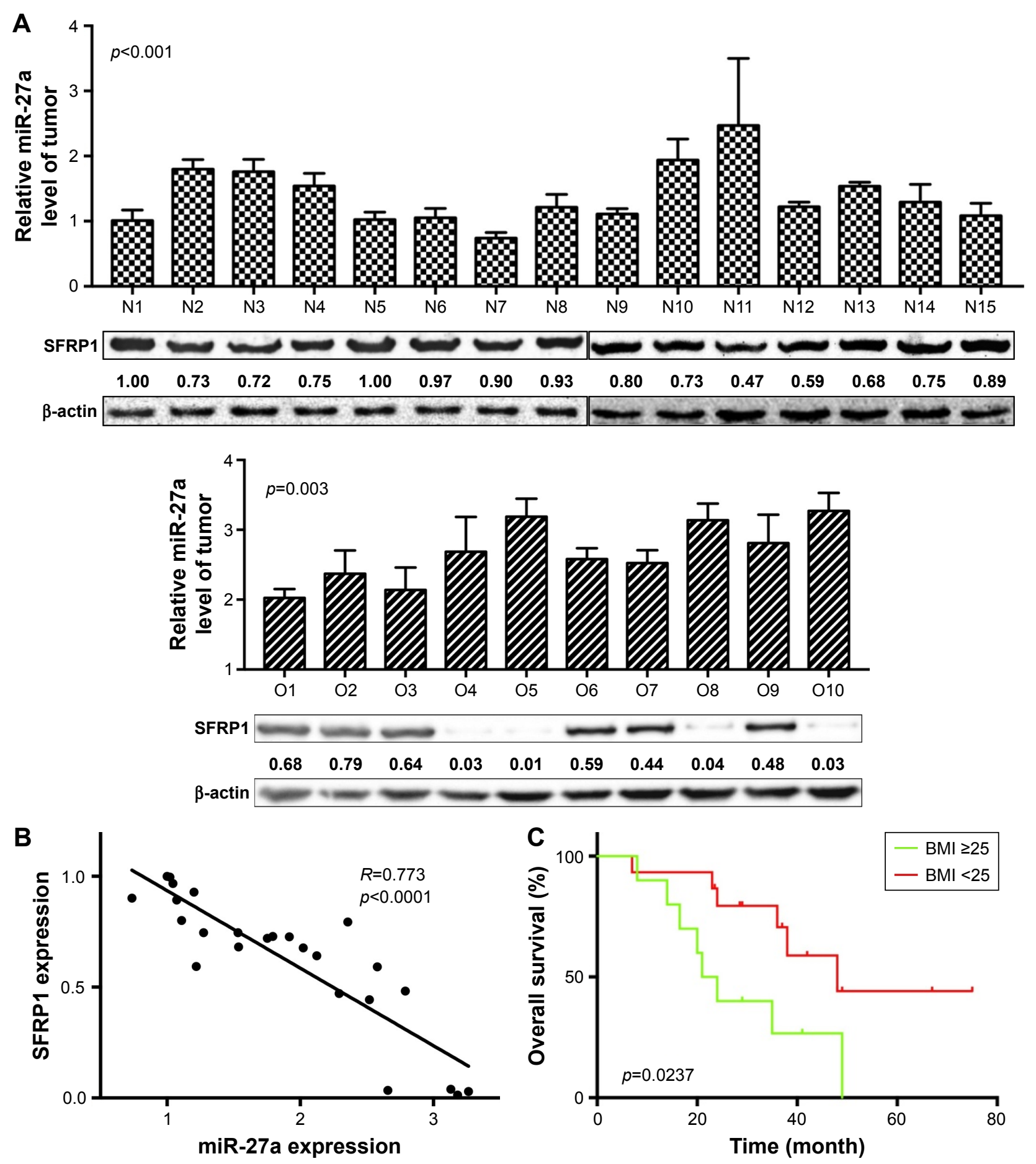

Figure 6 Expression of miR-27a and SFRPI in HCC tissues of patients.

Notes: (A) qRT-PCR analysis of miR-27a and Western blot analysis of SFRPI in HCC tissues. $\beta$-actin was used as an internal control. N represents normal group and $O$ represents obesity group. NI was the negative control. Each bar represents the mean \pm SD of 3 replicates. (B) Correlation analysis between miR-27a expression and SFRPI expression in HCC tissues. (C) Kaplan-Meier overall survival curves for patients in normal and obesity groups.

Abbreviations: BMI, body mass index; HCC, hepatocellular carcinoma; QRT-PCR, quantitative reverse transcription polymerase chain reaction; SFRPI, secreted frizzledrelated protein I.

molecules that influence carcinoma initiation and metastatic progression. ${ }^{25,26} \mathrm{miR}-27 \mathrm{a}$ has been linked with cancer progression, such as renal carcinoma, colon carcinoma, gastric adenocarcinoma, pancreatic cancer and lung cancer. ${ }^{27-31}$ Our previous work showed that miR-27a was overexpressed in obese HCC patients than patients with normal body weight in both serums and tissues. ${ }^{19}$ We repeated the work with 25 human samples and found that patients with obesity had high 
levels of miR-27a and poor clinical outcomes. In the present study, we cultured differentiated 3T3-L1 cells with HepG2 cells in vitro and set up HCC metastasis model in obesity mouse in vivo to mimic the tumor microenvironment in obese status. MiR-27a expression is consistently upregulated in culture medium of 3T3-L1 with transfection of miR-27a plasmid, as well as adipose tissue, liver, and serum in HCC metastasis model based on obesity. We found that secretory miR-27a promotes migration and invasion of HCC cells in vitro, and we also observed more visible metastasis nodes on lung tissue from orthotopic liver transplantation tumor in obese. Our results confirmed that miR-27a plays a critical role on HCC metastasis, particularly in obesity HCC patients.

Numerous signaling pathways are deregulated during the initiation and progression of $\mathrm{HCC}$, including Wnt/ $\beta$-catenin, JAK/STAT, PI3K/Akt/mTOR, Ras/Raf/MEK/ ERK, as well as Hedgehog pathways. ${ }^{32}$ It is reported that miR-27a promoted gastric cancer cell metastasis by activating $\mathrm{Wnt} / \beta$-catenin signaling pathway $^{33}$ and miR-27a regulated $\mathrm{Wnt} / \beta$-catenin signaling through directly targeting SFRP1 in glioma. ${ }^{24}$ SFRP1, which is located at chromosome 8 p12-11.1, is a $35-\mathrm{kDa}$ glycoprotein that acts as a member of negative regulators of Wnt. ${ }^{34}$ After it is released into the extracellular space, SFRP1 can block the activation of Wnt signaling pathway by competitively binding to the frizzled receptors. ${ }^{35}$ The epigenetic silencing of SFRP1 was observed in HCC and loss of SFRP1 expression is associated with poor prognosis. ${ }^{34,36,37}$ These studies provide a clue to investigate whether miR-27a promote HCC metastasis by targeting SFRP1, which is further involved in $\mathrm{Wnt} / \beta$-catenin pathway. To determine whether the miR-27a regulates HCC progression via SFRP1, we overexpressed SFRP1 in HepG2 cells, co-culturing with adipocyte 3T3-L1 transfected miR-27a plasmid. It was seen that the expressing of SFRP1 prevented the migration and invasion of HCC in vitro. Meanwhile, the obesity mouse model revealed upregulation of miR-27a accompanied by the loss of SFRP1 expression in HF group. The decreased SFRP1 expression was closely related with the elevated number of lung metastatic nodes in tumor metastasis model of obese mice. We further verified the inverse correlation between miR-27a and SFRP1 protein levels in human HCC tissues. In addition, the loss of SFRP1 resulted in Wnt proteins binding to the frizzled receptor, which led to $\beta$-catenin accumulation in the cytoplasm by escaping from degradation and translocated into the nucleus, where it interacted with its downstream transcription factors lymphoid-enhancing factor/T-cell factor. ${ }^{22}$ Our results showed that overexpressed miR-27a upregulated the protein level of $\beta$-catenin and MMP7; this effect was cut down by overexpression of SFRP1 in HCC cells. This tendency reappeared in tumor tissues of the HF group, compared with that of control group. Our study pointed that SFRP1 may act as a direct target gene of miR-27a and can negatively modulate Wnt/ $\beta$-catenin pathway in HCC metastasis progression.

The EMT is a crucial developmental event in embryonic tissue formation and regeneration and is also hijacked by carcinomas during solid tumor progression. ${ }^{38}$ It is characterized by loss of the epithelial homotypic adhesion molecule E-cadherin and the gain of mesenchymal markers, such as Vimentin, which promotes tumor invasion and metastasis. ${ }^{39}$ EMT is accompanied by the activation of $\mathrm{Wnt} / \beta$-catenin pathway. $\beta$-catenin is an essential molecule both in cadherinmediated cell adhesion and canonical Wnt signaling. Our results showed that the increased expression of Vimentin and the decreased E-cadherin expression was linked with high level of $\beta$-catenin in HF group, and the opposite tendency was observed in the control group. This suggested a possible link between obesity and EMT through Wnt/ $\beta$-catenin signaling, but the mechanism needs to be further investigated.

\section{Conclusion}

Our study suggested that obesity-associated miR-27a may promote HCC metastasis by suppressing SFRP1, subsequently activating $\mathrm{Wnt} / \beta$-catenin signaling, and to some extent, involvement of EMT. Our findings shed light on the mechanism the HCC metastasis in obese individuals and provided miR-27a as a promising target for liver cancer therapy in obese patients.

\section{Acknowledgments}

This work is sponsored by Natural Science Foundation of Jilin Province (20160101203JC), the Education Department of Jilin Province grant (2016, No 452), the Undergraduate Innovative Program of Jilin University (2016B79690, 2015741102), and Norman Bethune Program of Jilin University (2015224). Most of the experiments were carried out at the Nanomedicine Engineering Laboratory of Jilin Province.

\section{Disclosure}

The authors report no conflicts of interest in this work.

\section{References}

1. WHO [webpage on the Internet]. Obesity and overweight. Available from: http://www.who.int/mediacentre/factsheets/fs311/en/. Accessed October 5, 2016.

2. Canoy D, Buchan I. Challenges in obesity epidemiology. Obes Rev. 2007; 8(Suppl 1):1-11. 
3. Teoh SL, Das S. Tumour biology of obesity-related cancers: understanding the molecular concept for better diagnosis and treatment. Tumour Biol. 2016;37(11):14363-14380.

4. Sinicrope FA, Foster NR, Sargent DJ, O'Connell MJ, Rankin C. Obesity is an independent prognostic variable in colon cancer survivors. Clin Cancer Res. 2010;16(6):1884-1893.

5. Reeves GK, Pirie K, Beral V, et al. Million Women Study Collaboration. Cancer incidence and mortality in relation to body mass index in the Million Women Study: cohort study. BMJ. 2007;335(7630):1134

6. Carmichael AR. Obesity as a risk factor for development and poor prognosis of breast cancer. BJOG. 2006;113(10):1160-1166.

7. El-Serag HB, Rudolph KL. Hepatocellular carcinoma: epidemiology and molecular carcinogenesis. Gastroenterology. 2007;132(7): 2557-2576.

8. Maluccio M, Covey A. Recent progress in understanding, diagnosing, and treating hepatocellular carcinoma. CA Cancer J Clin. 2012;62(6): 394-399.

9. Huang ZY, Liang BY, Xiong M, et al. Long-term outcomes of repeat hepatic resection in patients with recurrent hepatocellular carcinoma and analysis of recurrent types and their prognosis: a single-center experience in China. Ann Surg Oncol. 2012;19(8):2515-2525.

10. Tang B, Qi G, Sun X, et al. HOXA7 plays a critical role in metastasis of liver cancer associated with activation of Snail. Mol Cancer. 2016; 15(1):57.

11. El-Serag HB. Hepatocellular carcinoma. N Engl J Med. 2011;365(12): 1118-1127.

12. Turati F, Talamini R, Pelucchi $\mathrm{C}$, et al. Metabolic syndrome and hepatocellular carcinoma risk. Br J Cancer. 2013;108(1):222-228.

13. Samanic C, Chow WH, Gridley G, Jarvholm B, Fraumeni JF Jr. Relation of body mass index to cancer risk in 362,552 Swedish men. Cancer Causes Control. 2006;17(7):901-909.

14. Borena W, Strohmaier S, Lukanova A, et al. Metabolic risk factors and primary liver cancer in a prospective study of 578,700 adults. Int J Cancer. 2012;131(1):193-200.

15. Schlesinger S, Aleksandrova K, Pischon T, et al. Abdominal obesity, weight gain during adulthood and risk of liver and biliary tract cancer in a European cohort. Int J Cancer. 2013;132(3):645-657.

16. Marengo A, Rosso C, Bugianesi E. Liver cancer: connections with obesity, fatty liver, and cirrhosis. Annu Rev Med. 2016;67:103-117.

17. Karagozian R, Derdak Z, Baffy G. Obesity-associated mechanisms of hepatocarcinogenesis. Metabolism. 2014;63(5):607-617.

18. Vanni E, Bugianesi E. Obesity and liver cancer. Clin Liver Dis. 2014; 18(1):191-203.

19. Sun B, Li J, Shao D, et al. Adipose tissue-secreted miR-27a promotes liver cancer by targeting FOXO1 in obese individuals. Onco Targets Ther. 2015;8:735-744.

20. Li S, Li J, Fei BY, et al. MiR-27a promotes hepatocellular carcinoma cell proliferation through suppression of its target gene peroxisome proliferator-activated receptor $\gamma$. Chin Med J (Engl). 2015;128(7): 941-947.

21. WHO Expert Consultation. Appropriate body-mass index for Asian populations and its implications for policy and intervention strategies. Lancet. 2004;363(9403):157-163.

22. Vilchez V, Turcios L, Marti F, Gedaly R. Targeting Wnt/beta-catenin pathway in hepatocellular carcinoma treatment. World J Gastroenterol. 2016;22(2):823-832.
23. Guo D, Li Q, Lv Q, Wei Q, Cao S, Gu J. MiR-27a targets sFRP1 in $\mathrm{hFOB}$ cells to regulate proliferation, apoptosis and differentiation. PLoS One. 2014;9(3):e91354.

24. Wang K, Xie D, Xie J, et al. MiR-27a regulates Wnt/beta-catenin signaling through targeting SFRP1 in glioma. Neuroreport. 2015;26(12): 695-702.

25. Ferrante SC, Nadler EP, Pillai DK, et al. Adipocyte-derived exosomal miRNAs: a novel mechanism for obesity-related disease. Pediatr Res. 2015;77(3):447-454.

26. Kloting N, Berthold S, Kovacs P, et al. MicroRNA expression in human omental and subcutaneous adipose tissue. PLoS One. 2009;4(3): e4699.

27. Peng H, Wang X, Zhang P, Sun T, Ren X, Xia Z. miR-27a promotes cell proliferation and metastasis in renal cell carcinoma. Int J Clin Exp Pathol. 2015;8(2):2259-2266.

28. Gao Y, Li BD, Liu YG. Effect of miR27a on proliferation and invasion in colonic cancer cells. Asian Pac J Cancer Prev. 2013;14(8): $4675-4678$

29. Liu T, Tang H, Lang Y, Liu M, Li X. MicroRNA-27a functions as an oncogene in gastric adenocarcinoma by targeting prohibitin. Cancer Lett. 2009;273(2):233-242.

30. Xia J, Cheng L, Mei C, et al. Genistein inhibits cell growth and invasion through regulation of miR-27a in pancreatic cancer cells. Curr Pharm Des. 2014;20(33):5348-5353.

31. Acunzo M, Romano G, Palmieri D, et al. Cross-talk between MET and EGFR in non-small cell lung cancer involves miR-27a and Sprouty2. Proc Natl Acad Sci U S A. 2013;110(21):8573-8578.

32. Hoshida Y, Toffanin S, Lachenmayer A, Villanueva A, Minguez B, Llovet JM. Molecular classification and novel targets in hepatocellular carcinoma: recent advancements. Semin Liver Dis. 2010;30(1):35-51.

33. Zhang Z, Liu S, Shi R, Zhao G. miR-27 promotes human gastric cancer cell metastasis by inducing epithelial-to-mesenchymal transition. Cancer Genet. 2011;204(9):486-491.

34. Davaadorj M, Imura S, Saito YU, et al. Loss of SFRP1 expression is associated with poor prognosis in hepatocellular carcinoma. Anticancer Res. 2016;36(2):659-664.

35. Bafico A, Gazit A, Pramila T, Finch PW, Yaniv A, Aaronson SA. Interaction of frizzled related protein (FRP) with Wnt ligands and the frizzled receptor suggests alternative mechanisms for FRP inhibition of Wnt signaling. J Biol Chem. 1999;274(23):16180-16187.

36. Xie Q, Chen L, Shan X, et al. Epigenetic silencing of SFRP1 and SFRP5 by hepatitis $\mathrm{B}$ virus $\mathrm{X}$ protein enhances hepatoma cell tumorigenicity through Wnt signaling pathway. Int J Cancer. 2014;135(3):635-646.

37. Kaur P, Mani S, Cros MP, et al. Epigenetic silencing of sFRP1 activates the canonical Wnt pathway and contributes to increased cell growth and proliferation in hepatocellular carcinoma. Tumour Biol. 2012;33(2): 325-336.

38. Steinway SN, Zanudo JG, Ding W, et al. Network modeling of TGFbeta signaling in hepatocellular carcinoma epithelial-to-mesenchymal transition reveals joint sonic hedgehog and Wnt pathway activation. Cancer Res. 2014;74(21):5963-5977.

39. Thiery JP, Acloque H, Huang RY, Nieto MA. Epithelial-mesenchymal transitions in development and disease. Cell. 2009;139(5):871-890. 


\section{Supplementary material}
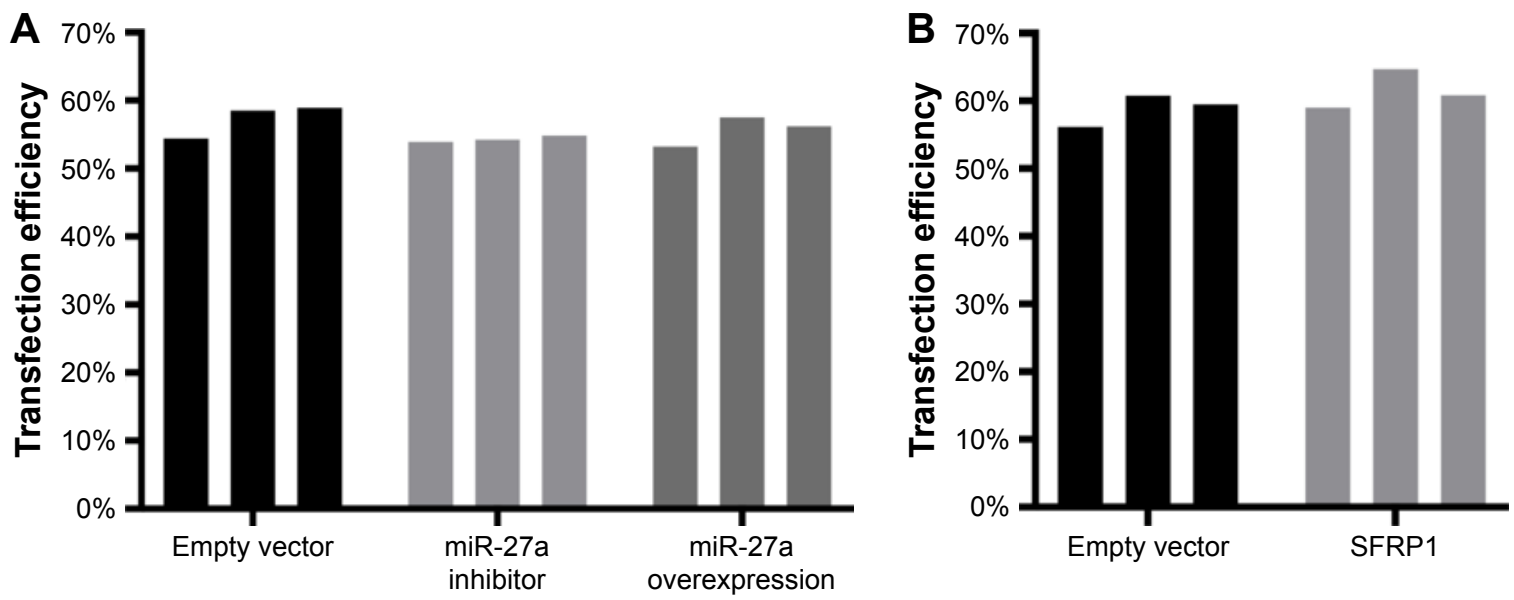

Figure SI FACS analysis of transfection efficacy.

Notes: (A) 3T3-LI cells transfected with the empty vectors, the miR-27a inhibitor plasmids, and the miR-27a expression plasmids. (B) HepG2 cells transfected with the empty vectors and the miR-27a expression plasmids. Each transfection experiment was performed 3 times.

Abbreviations: FACS, fluorescence activated cell sorting; SFRPI, secreted frizzled-related protein I.

\section{Publish your work in this journal}

OncoTargets and Therapy is an international, peer-reviewed, open access journal focusing on the pathological basis of all cancers, potential targets for therapy and treatment protocols employed to improve the management of cancer patients. The journal also focuses on the impact of management programs and new therapeutic agents and protocols on patient perspectives such as quality of life, adherence and satisfaction. The manuscript management system is completely online and includes a very quick and fair peer-review system, which is all easy to use. Visit http://www.dovepress.com/testimonials.php to read real quotes from published authors. 